Technological University Dublin

ARROW@TU Dublin

\title{
Analysis of Wind Velocity Signals for Estimating the Wave Power Density of Ireland's Coastal Resource
}

Jonathan Blackledge

Technological University Dublin, jonathan.blackledge@tudublin.ie

Eugene Coyle

Technological University Dublin

Derek Kearney

Technological University Dublin, derek.kearney@tudublin.ie

See next page for additional authors

Follow this and additional works at: https://arrow.tudublin.ie/engscheleart2

Part of the Engineering Commons

\section{Recommended Citation}

Blackledge, J., Coyle, E., Kearney, D., McGuirk, R.:Analysis of Wind Velocity Signals for Estimating the Wave Power Density of Ireland's Coastal Resource. Submitted for Irish Signals and Systems Conference 2012, NUI Maynooth, 2012. doi:10.1049/ic.2012.0176

This Conference Paper is brought to you for free and open access by the School of Electrical and Electronic Engineering at ARROW@TU Dublin. It has been accepted for inclusion in Articles by an authorized administrator of ARROW@TU Dublin. For more information, please contact arrow.admin@tudublin.ie, aisling.coyne@tudublin.ie,gerard.connolly@tudublin.ie.

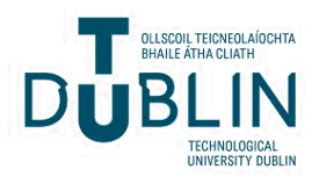


Authors

Jonathan Blackledge, Eugene Coyle, Derek Kearney, and Ronan McGuirk

This conference paper is available at ARROW@TU Dublin: https://arrow.tudublin.ie/engscheleart2/49 


\title{
Analysis of Wind Velocity Signals for Estimating the Wave Power Density of Ireland's Coastal Resource
}

\author{
Jonathan Blackledge, Eugene Coyle, Ronan McGuirk and Derek Kearney
}

\author{
Dublin Energy Laboratory \\ Dublin Institute of Technology \\ Kevin Street, Dublin 8, Ireland
}

\begin{abstract}
The aim of this paper is to report on a possible correlation between the Lévy index for the wind velocity and the mean Energy Density of sea surface waves in the same location. The result is based on data obtained from six buoys located around the coast of Ireland and maintained by the Marine Institute of Ireland. These buoys provide signals on the wind velocity, wave height and wave period as well as other data on an hourly interval. Using this data, we consider the relationship between a stochastic model for the time variations in wave height that, in turn, is based on a non-Gaussian model for the wind force characterized by the Lévy index. Although the results presented in this paper can not be said to statistically significant due to the limited amount of data that is available (i.e. the Marne Institute of Ireland only maintains six buoys around the coast of Ireland) they indicate the possibility of developing a method of accurately estimating the energy and power densities of sea waves from knowledge of the wind velocity alone.
\end{abstract}

Keywords - Wave Energy Density, wind velocity, Lévy index, non-Gaussian wind velocity model, sea surface temporal wave model

\section{INTRODUCTION}

Ocean wave energy [1] has significant potential in terms of the contributions it can make to the development of a renewable energy resource [2]. Generating electricity from open sea surface waves has negligible environmental impact, increases the amount of electricity that originates from renewable energy technologies, minimizes the use of fossil fuels in the long run and addresses climate change problems. One of the principal problems associated with ocean wave energy is how to make generation of the energy economically viable. Part of this 'viability exercise' includes the development of physical and engineering models to accurately predict the energy and power available from open sea surface wave patterns. It is in the context of this problem that the results discussed in this paper are presented.

Ocean wave power is a concentrated form of solar energy. Winds generated by the differential heating of the earth pass over open bodies of water and push surface water particles along with it, setting up a rolling motion in the water and moving the water particles in a vertical and circular path. The energy and power densities of a wave are proportional to the square of the wave amplitude and knowledge of the average wave height is therefore important when considering where to place a wave farm. Figure 1 shows an average wave height map of the world where it is clear that the northern hemisphere (i.e. the northern Atlantic and Pacific oceans) have the largest average wave heights. Further, in terms of the propensity of these waves to coast lines, there are two principal regions that stand out: (i) the Aleutian Islands; (ii) the west coasts of Ireland and Scotland. However, the latter coasts are regions with a higher population density and easier access to the infrastructure required to exploit wave energy technology. On the west coast of Ireland, wave heights can vary from 2-12 metres over a week depending on seasonal changes.

The motion of sea surface waves (generated by the wind) is principally determined by the wind speed and, in particular, the gradient of the wind velocity which induces a force. Thus, understand- 


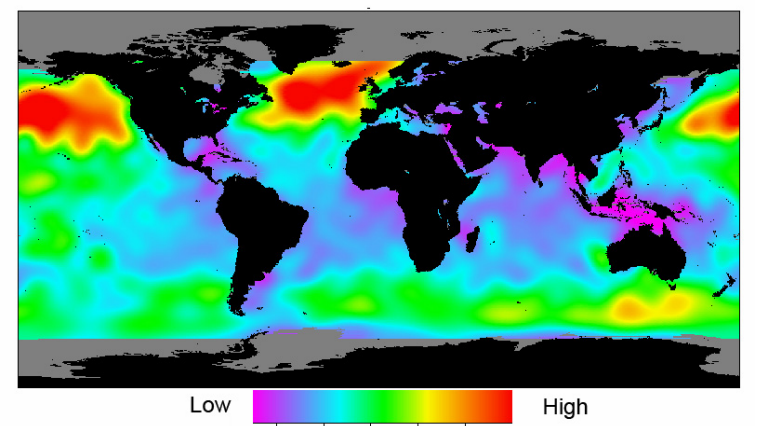

Fig. 1: World map of average wave heights.

ing the temporal and spatial variations of the wind force regulated through different angles of incidence upon the sea surface is a fundamental issue. Another issue is the characteristic spectrum over which the wind force is converted into wave motion. Because it is not possible to uniquely simulate such complex interactions on an entirely deterministic basis (e.g. the wind velocity can not be known precisely as a function of time) over large scales, we consider stochastic models to investigate a correlation between the energy associated with a sea surface wave stream and the wind velocity time series characterised by the Lévy index.

In order to validate a stochastic model two approaches can be considered. The first is to use Computational Fluid Dynamics (CFD) to simulate sea surface waves over relatively small scale surface areas in order to confirm the statistical validity or otherwise of the stochastic models developed. The second and complementary method is to consider the statistical compatibility of the stochastic model with known data (e.g. wave height time series) which will inevitably be of a limit extent and validity (the use of CFD thereby being required). In this paper, we address the second approach using data obtained from the Irish Marine Weather Buoy Network [3].

The ultimate aim of this work is to consider a model for time varying sea surface wave patterns generated by a wind velocity field that is compatible with known data for a given location. The purpose of this is to derive more precise estimates of the expected power output from a given wave energy converter with known performance characteristics. The approach provides the potential to identify the optimal location of a wave farm from knowledge of the wind velocity alone. In this paper we provide evidence for a possible correlation between the expected wave Energy Density and the Lévy index for the wind velocity.

\section{Linear WaVe Models}

Linear wave spectrum models assume that the distance over which the waves develop and the dura- tion over which the wind blows are sufficient for the waves to achieve their maximum energy for the given wind speed. It is assumed that waves can be represented by sinusoidal forms. This relies on the following: (i) Waves vary in a regular way around an average wave height; (ii) there are no energy losses due to friction or turbulence, for example; (iii) the wave height is much smaller than the wavelength. These principal assumptions provide the basis for predicting wave amplitudes on a statistical basis and it is upon this basis that many wave energy converters are designed in which the wave amplitude is taken to conform to a Rayleigh distribution. However, this distribution is known to be inaccurate which is primarily due to a lack of understanding of how, on a statistical basis, wind energy is converted into wave energy. From a statistical point of view, what is required is a physical model that can accurately predict the distribution of sea surface waves given knowledge on the distribution of the wind velocity. A solution to this problem could then be used to estimate the 'quality of power' from a wave farm given statistical parameters that reflect the environmental conditions in which the wave farm is operating. The quality of power is based on measures that include the energy and power densities of the ocean surface. These measures are discussed in the following section.

\section{Energy And Power Density Measures}

The Energy Density (energy in Joules per unit area) of a continuous sea surface wave can be obtained by considering the oscillation of a column of water perpendicular to the plane. Let $h$ be the height of the column with area $A$ above the plane at $h=0$. Denoting the density of water by $\rho \simeq 1000 \mathrm{kgm}^{-3}$ and with $g=9.81 \mathrm{~ms}^{-2}$ representing the acceleration due to gravity, the downward force is give by $F=h A \rho g$. Thus we can write (from Newton second law) the time dependent wave equation

$$
\frac{d^{2} h}{d t^{2}}=-\omega^{2} h, \quad \omega^{2}=\frac{A \rho g}{m}
$$

whose general solution is given by

$$
h(t)=a \exp ( \pm i \omega t), \quad \omega=\sqrt{\frac{A \rho g}{m}}
$$

where $\omega$ is the angular frequency and $a$ is the amplitude of the wave. The energy is then (from Newton's energy formula)

$$
E=\frac{1}{2} m|v|^{2}=\frac{1}{2} m\left|\frac{d h}{d t}\right|^{2}=\frac{1}{2} A \rho g a^{2}
$$

giving an expression for the Energy Density $E$ := $E / A$ of the form

$$
E=\frac{1}{8} \rho g H^{2} \quad \mathrm{Jm}^{-2}
$$




$$
\simeq 1.23 H^{2} \mathrm{kJm}^{-2}
$$

where $H=2 a$ is the wave height in metres. The Power Density is then given by

$$
P=\frac{E}{T} \quad \mathrm{Wm}^{-2}
$$

where $T$ is the period of the wave in seconds.

This result refers to the simplistic case of a continuous wave oscillating at a single (angular) frequency $\omega$. It also assumes linearity where the wave amplitude is taken to have relatively small amplitudes. In reality the time signature of a surface wave stream measured at a spatial location on the sea surface will be composed of a range of amplitudes that vary in time reflecting a characteristic frequency spectrum. In this case, we can consider $H$ to be given by the mean or root-mean-square of the wave stream. Similarly the period of the waves will vary and we may consider $T$ to be the mean of periods. Thus, if we consider an average wave height of say 1 metre, then the Energy Density is $1.23 \mathrm{kJm}^{-2}$ giving a Power Density of $1 \mathrm{kWm}^{-2}$ for a mean wave period of 1 second.

A further measure of a wave energy resource is the power per meter of the wave front (wave crest) or Energy Flux. This measure can be calculated by multiplying the Energy Density by the wave front or group velocity to give

$$
F \simeq 0.5 H^{2} T, \mathrm{kWm}^{-1}
$$

\section{Analaysis of Sample Data From the Irish Marine Weather Buoy Network}

The Irish Marine Weather Buoy Network [3] maintains six weather buoys that record data on the Atmospheric Pressure (mb), Wind Direction (Degrees), Wind Speed (in Knots where $1 \mathrm{Knot}=0.514444 \mathrm{~m} / \mathrm{s})$, Gust(Knots), Dry Bulb Temperature $\left({ }^{\circ} \mathrm{C}\right)$, Dew Point $\left.\left({ }^{\circ} \mathrm{C}\right)\right)$, Sea Temperature $\left({ }^{\circ} \mathrm{C}\right)$, Wave Period (seconds), Wave Height (metres) and Relative Humidity (\%). The buoys (M1-M6) are located as given in Figure 2

Figure 3 shows an example of data taken from the M1 buoy which is located off the west cost of Ireland (see Figure 2) at position $53.1266^{\circ} \mathrm{N}$ $11.2000^{\circ} W$. This Figure shows a signal consisting of $\sim 8000$ samples of hourly data on the wind velocity in metres per second, the wave height in metres and the wave period in seconds The data available from [3] is not always contiguous as some samples or sequences of samples are the product of null returns. The data therefore needs to be filtered to eradicate any null entries. In cases when different data streams are being used on a comparative basis, as in Figure 3, each data stream needs to filtered consecutively eradicating all data points in all arrays groups when ever a single null return is detecting in any single array. Thus, the signals

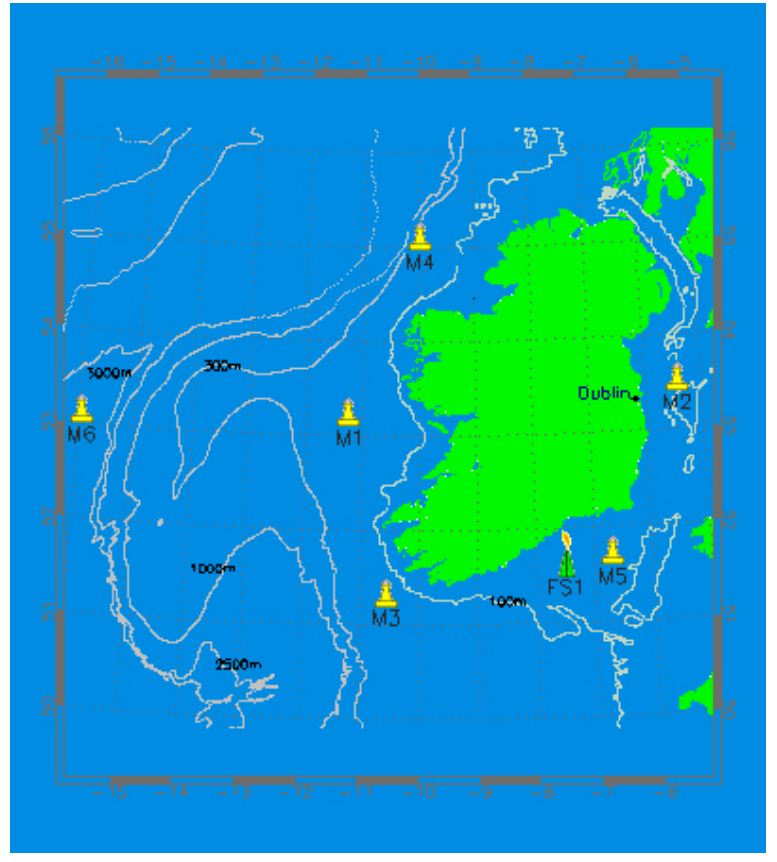

Fig. 2: Location of the Irish Marine Weather Buoys M1 M6.
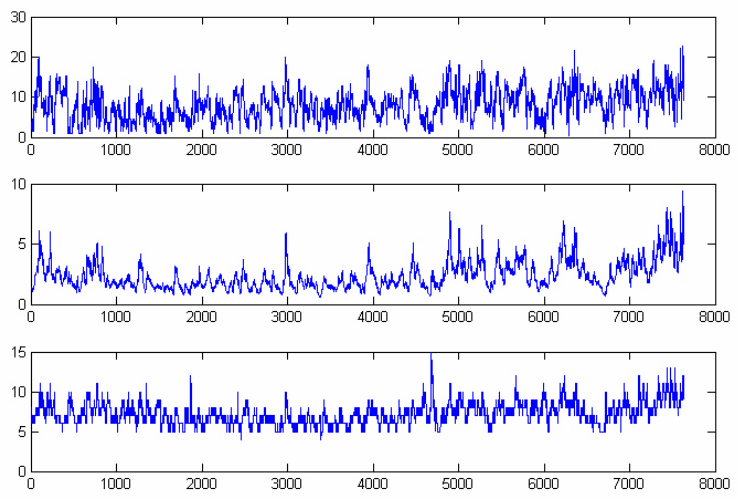

Fig. 3: Time series of the wind velocity in $\mathrm{m} / \mathrm{s}$ (top) wave height in metres (centre) and the wave period in seconds (bottom) - hourly sampling.

given in Figure 3 do not represent a contiguous stream of data output from the M1 buoy but a null entry filtered version of the data. However, this does not effect the statistical characteristics of the data, and, in each case, the data is seen to be Rayleigh distributed as illustrated in Figure 4 which shows 100-bin histograms of the signals given in Figure 3. Figure 5 shows plots of the Energy Density, the Power Density and the Energy Flux computed from equations (1), (2) and (3), respectively, using the samples of data from the M1 buoy shown in Figure 3. Note that all three signals have similar time signatures, and, apart from scaling, their statistical characteristics are similar. However, the signals are significantly erratic with Energy Fluxes, for example, ranging from long periods of a few $\mathrm{kW} / \mathrm{m}$ to short periods in excess of 

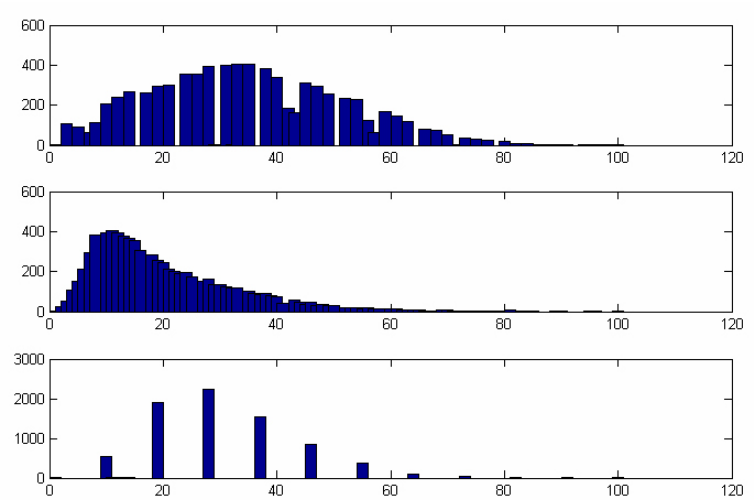

Fig. 4: 100-bin histograms of data on the wind velocity (top), the wave height (centre) and the wave period (bottom) as given in Figure 3 .

$300 \mathrm{~kW} / \mathrm{m}$. Data of this type can be acquired from
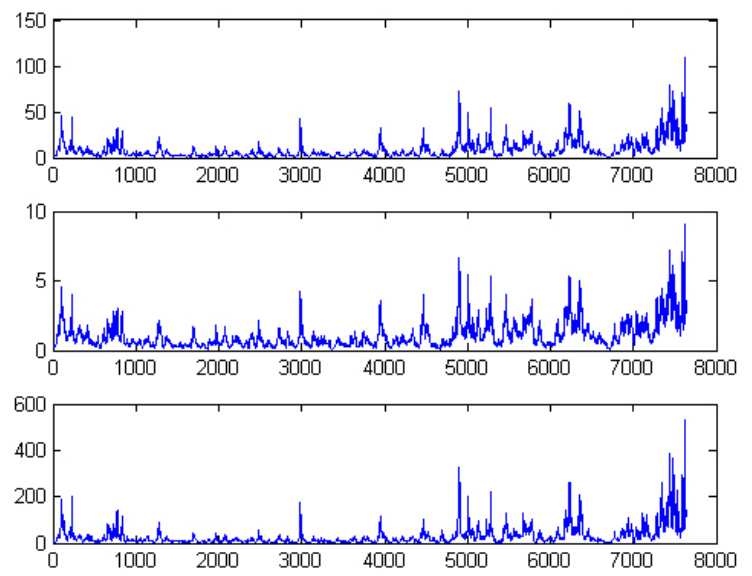

Fig. 5: Plots of the Energy Density in $\mathrm{kJ} / \mathrm{m}^{2}$ (top), Power Density in $\mathrm{W} / \mathrm{m}^{2}$ (centre) and the Energy Flux in $\mathrm{kW} / \mathrm{m}$ (bottom) using data obtained for the M1 Marine Institute buoy. The sampling rate is hourly.

all six Marine Institute buoys. We consider an evaluation of the energy/power parameters given by equations (1)-(3) and the wind velocity. The results are shown in Figure 6 which gives scatter plots of the mean wind velocity for the mean values of the Energy Density, the Power Density and the Energy Flux using filtered time series of the type given in Figure 3 for buoys M1 to M6 as tabulated in Table 1.

From this Figure, we observe the following: (i) The relationship between the mean wind velocity and the mean values of the Energy Density, the Power Density and the Energy Flux is similar in all cases; (ii) there is no clear scaling relationship between the mean wind velocity and these parameters. It is in this context that we now investigate a possible scaling relationship between the Energy Flux and the Levy index for the wind velocity using models discussed in the following section.

\begin{tabular}{|c|c|c|c|c|}
\hline Buoy & $\begin{array}{c}\text { Wind } \\
\text { Velocity } \\
\mathrm{m} / \mathrm{s}\end{array}$ & $\begin{array}{c}\text { Energy } \\
\text { Denity } \\
\mathrm{kJ} / \mathrm{m}^{2}\end{array}$ & $\begin{array}{c}\text { Power } \\
\text { Density } \\
\mathrm{W} / \mathrm{m}^{2}\end{array}$ & $\begin{array}{c}\text { Energy } \\
\text { Flux } \\
\mathrm{kW} / \mathrm{m}\end{array}$ \\
\hline M1 & 8.1308 & 8.5008 & 1.0611 & 28.7169 \\
M2 & 6.3215 & 2.5244 & 0.4722 & 5.6346 \\
M3 & 7.5744 & 11.8143 & 1.4957 & 39.5510 \\
M4 & 7.2813 & 4.8766 & 0.6600 & 15.2146 \\
M5 & 8.3263 & 4.8171 & 0.7789 & 12.7181 \\
M6 & 8.0778 & 19.4454 & 2.3788 & 66.9620 \\
\hline
\end{tabular}

Table 1: Tabulation of the mean value parameters computed from the time series data obtained from the Marine Institute buoys M1-M6.
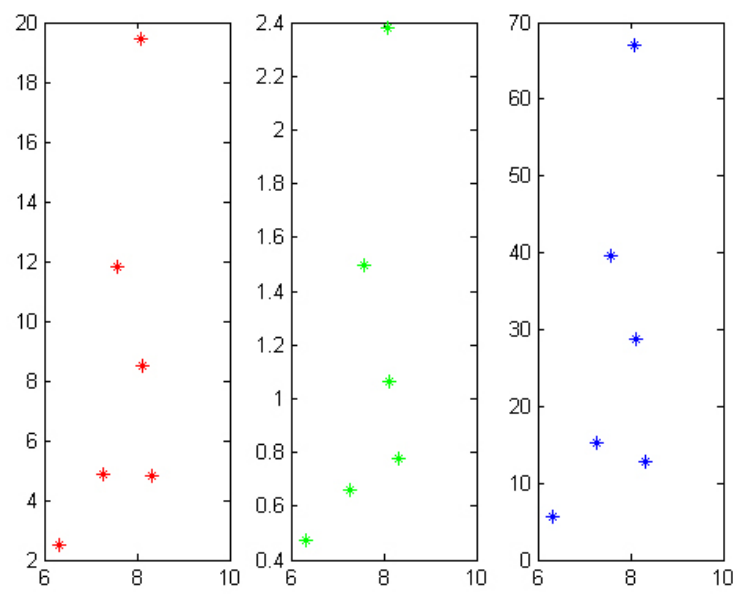

Fig. 6: Scatter plots of the mean wind velocity in $\mathrm{m} / \mathrm{s}$ versus the mean Energy Density in $\mathrm{kJ} / \mathrm{m}^{2}$ (left), mean Power Density in $\mathrm{W} / \mathrm{m}^{2}$ (centre) and the mean Energy Flux in $\mathrm{kW} / \mathrm{m}$ (right) for data obtained from the six Marine Institute buoys given in Figure 2.

\section{A Scaling Relationship for Sea Surface WAVES}

We consider the model derived in [4] for time varying sea surface wave which is compounded in the following result:

$$
u(t)=\frac{\Omega}{\pi} \operatorname{sinc}(\Omega t) \otimes f(t)
$$

where $\otimes$ denotes the convolution integral over time $t, u(t)$ is the wave amplitude, $f(t)$ is the force generated by the wind as a function of time and $\Omega$ is the bandwidth that is characteristic of the conversion of the force of the wind on the surface of the sea into wave motion. Equation (4) is based on the following limiting observations: (i) the sea surface consists of a spectrum of two-dimensional waves oscillating at different frequencies; (ii) all sea surface waves have relatively low frequencies $\sim 1 \mathrm{~Hz}$ or less; (iii) the waves are not correlated in the sense that the wave pattern is locally dependent on the interaction of the wind with the sea surface over a limited area particularly in the higher frequency range. Point (iii) also means that any attenuation 
of the surface due to the viscosity of sea water can also be neglected.

For unit mass, the wind force is given by the gradient of the wind velocity. In [5] and [6], a model for the wind velocity is considered that is based on a Lévy distribution for the wind force. It is shown that this approach leads to a stochastic model for the wind velocity $v(t)$ given by

$$
v(t)=\frac{1}{\Gamma(1 / \gamma) t^{1-1 / \gamma}} \otimes n(t)
$$

so that (for unit mass) the wind force is then given by

$$
f(t)=\frac{1}{\Gamma(1 / \gamma) t^{1-1 / \gamma}} \otimes n^{\prime}(t)
$$

where $\Gamma(1 / \gamma)$ is the Gamma function, $n(t) \in[0,1]$ is a 'white noise' source, $n^{\prime}(t)$ denotes the differential of $n(t)$ and $\gamma \in(0,2]$ is the Lévy index. Given equation (6), equation (4) can be written in the form

$$
u(t)=\frac{\Omega}{\pi}\left(\frac{1}{\gamma}-1\right) \operatorname{sinc}(\Omega t) \otimes n(t) \otimes t^{\frac{1}{\gamma}-2}
$$

Using Euclidean norms we can then consider the inequality

$$
\|u(t)\|_{2} \leq A\left(\frac{1}{\gamma}-1\right)
$$

where

$$
A=\frac{\Omega}{\pi}\|\operatorname{sinc}(\Omega t)\|_{2} \times\|n(t)\|_{2} \times\left\|t^{2 / \gamma-4}\right\|_{2}
$$

and

$$
\|x(t)\|_{2} \equiv\left(\int|x(t)|^{2} d t\right)^{\frac{1}{2}}
$$

Thus, for $u(t) \exists \forall t \in[0, T]$, we can write

$$
\bar{u} \equiv \frac{1}{T} \int_{0}^{T}|u(t)|^{2} d t \leq \alpha \beta
$$

where

$$
\alpha=\left(\frac{1}{\gamma}-1\right)^{2}
$$

and $\beta=A^{2} / T$. It is now evident that the scaling relationship between the mean square amplitude $\bar{u}$ and the ' $\alpha$-index' as defined by equation (6) is determined by

$$
\ln \bar{u}=m \ln \alpha+c
$$

for some gradient $m$ and constant $c$. This relationship is investigated in the following section using data from the Marine Institute buoys M1-M6.

\begin{tabular}{|c|c|}
\hline Buoy & Lévy Index \\
\hline M1 & 1.5701 \\
M2 & 1.6125 \\
M3 & 1.5625 \\
M4 & 1.6078 \\
M5 & 1.6171 \\
M6 & 1.5751 \\
\hline
\end{tabular}

Table 2: Tabulation of Lévy index for sample wind velocity recorded by the Marine Institute buoys M1-M6.

\section{Power Spectrum Analysis using Wind VELOCITY DATA}

Equation (5) represents a model for the wind velocity which is characterized by the Lévy index $\gamma$. In this section, we determine the validity of the scaling relationship in equation (7) by computing the Lévy index for the wind velocity available from the Marine Institute Buoys, data that is similar to that given in Figure 3. To obtain an estimate of the Lévy index for a time series on the wind velocity, we use the method developed in [5]. This is based on a power spectrum analysis of equation (5) since, upon Fourier transform of equation (5), we can write

$$
V(\omega)=\frac{N(\omega)}{|\omega|^{\frac{1}{\gamma}}}
$$

where $V(\omega)$ and $N(\omega)$ are the Fourier transforms of $v(t)$ and $n(t)$ respectively. For a white noise source, the expected Power Spectral Density Function is given by

$$
P(\omega)=\frac{C}{|\omega|^{\frac{2}{\gamma}}}
$$

or

$$
\ln P(\omega)=\ln C+\frac{2}{\gamma} \ln |\omega|
$$

for some scaling constant $C$. Thus by applying a data fitting algorithm to the logarithm of the Power Spectrum of the wind velocity data, an estimate of $\gamma$ is obtained. For the same wind velocity data used to determine the parameters given in Table 1, we obtain the results given in Table 2 using the Orthogonal Linear Regression (OLR) method given in [7]

Figure 7 shows a logarithmic fit for the data on the Energy Density given in Table 1 and the and the $\alpha$-index computed from the Lévy indices given in Table 2 after sorting the data into ascending order. Using the OLR method discussed in [7] it is found that $m=15.7437$ and $c=33.0808$. Although this result does not represent a statistically significant outcome (due to the limited number of buoys maintained by the Marine Institute, i.e. the 6 buoys M1-M6), it is an indication of a possible correlation between the Lévy index for the local 
wind velocity and the corresponding Energy Density of the wave patterns for the same area.

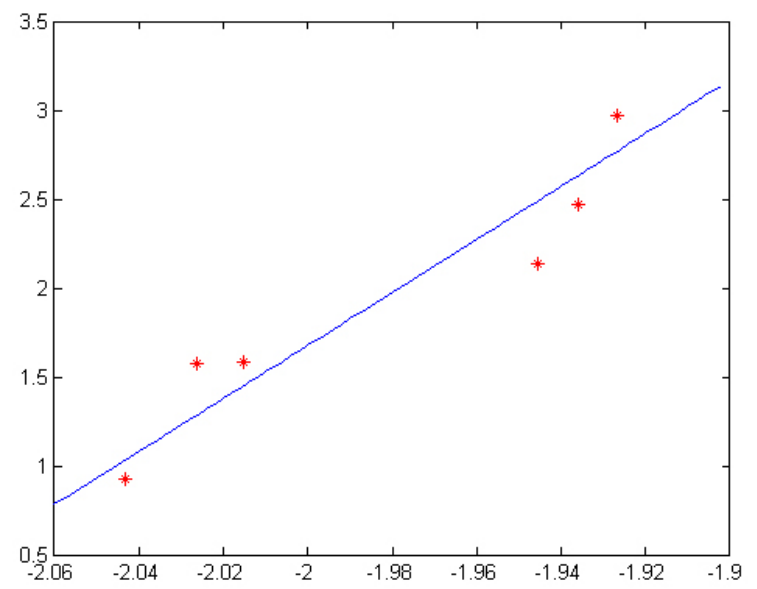

Fig. 7: A plot of the logarithm of the Energy Density versus the logarithm of $\alpha$ obtained from the data tabulated in Tables 1 and 2 (red point stars) and a best fit (solid blue line) to the data using equation (7) for $m=15.7437$ and $c=33.0808$ obtained using the OLR method.

\section{Conclusions and Future Work}

The results given in Figure 7 is an indication of an approach that could provide a way of estimating the mean Energy Density of a region of the sea surface from knowledge of the wind velocity (of the same region) alone. However, it is important to stress that the current result is not statistically significant due to the limited amount of data that is currently available (i.e. only data from 6 buoys has been available for this study). Moreover, the data is only available on an hourly sampled intervals and includes null returns that need to be filtered. However, the stochastic models used in this study provide a statistical measure that is not dependent on the sampling rate. This is because the wind velocity model is scale invariant, i.e. one of the fundamental properties of equation (5) is that

$$
\operatorname{Pr}[v(\lambda t)]=\lambda^{\frac{1}{\gamma}} \operatorname{Pr}[v(t)]
$$

for a scaling factor $\lambda$. The wind velocity is therefore taken to be a stochastic self-affine field (a random scaling fractal) whose statistical properties are the same (accept for scaling) at different sampling rates.

Coupled with the theoretical scaling law compounded in equation (7) and tentatively validated in Figure 7, the results provide the potential for developing a computational procedure to predict the mean Energy Density of a sea surface area from data obtained on the wind velocity alone. This has a number of practical advantages in the assessment of regions suitable for the exploitation of off-shore wave energy. Future work must include the validation (or otherwise) of the results presented in this paper. Future applications could include use of the approach to estimate the expected supplementary energy available by developing wave farms in the same regions as the off-shore wind farms being planned by the European Wind Energy Association (EWEA) for the 2030 offshore super-grid.

\section{ACKNOWLEDGEMENTS}

Jonathan Blackledge is supported by the Science Foundation Ireland Stokes Professorship Programme. The authors would like to thank the School of Electrical Engineering Systems and the Dublin Energy Lab for their support.

\section{REFERENCES}

[1] J. Cruz (Ed.), "Ocean Wave Energy: Current Status and Future Perspectives", Springer, 2008, ISBN 978-3-540-74894-6.

[2] "Renewable Energy Focus Handbook", Elsevier and Academic Press, 2009 ISBN: 978-0-12-374705-1, e-book available at: http://www.elsevierdirect.com/9780123747051

[3] "Irish Marine Weather Buoy Network", Marine Institute, Ireland, http://www.marine.ie/ home/publicationsdata/data/buoys/

[4] J. M. Blackledge, "A Generalized Model for the Evolution of Low Frequency Freak Waves", IANEG International Journal of Applied Mathematics, Vol. 41, Issue 1, 33-55, 2010 .

[5] J. M. Blackledge, E. Coyle and D. Kearney, "Non-Gaussian Analysis of Wind Velocity Data for the Determination of Power Quality Control", ISAST Trans. On Computing and Intelligent Systems, Vol. 3, No. 1, 78-86, 2011.

[6] J. M. Blackledge, E. Coyle and D. Kearney, "A Stochastic Model for Wind Turbine Power Quality using a Levy Index Analysis of Wind Velocity Data", The Third International Conference on Resource Intensive Applications and Services, INTENSIVE 2011, May 22-27, Venice, Italy, ISBN: 978-1-61208-006-2, 10-15, 2011.

[7] J. M. Blackledge, "Application of the Fractal Market Hypothesis for Modelling Macroeconomic Time Series", ISAST Transactions on Electronics and Signal Processing Vol. 2, No 1, 89-110, 2008. 